\title{
Infected bone resection plus adjuvant antibiotic-impregnated calcium sulfate versus infected bone resection alone in the treatment of diabetic forefoot osteomyelitis
}

Cheng-He Qin ${ }^{1 *+}$ (D), Chun-Hao Zhou ${ }^{2+}$, Hui-Juan Song ${ }^{3}$, Guo-Yun Cheng ${ }^{2}$, Hong-An Zhang ${ }^{2}$, Jia Fang ${ }^{1}$ and Rui Tao ${ }^{2}$

\begin{abstract}
Background: Managing with diabetic foot osteomyelitis (DFO) is challenging. Even after infective bone resection and thorough debridement, DFO is still difficult to cure and has a high recurrence rate. This retrospective study aims to compare the outcomes of two treatment methods, infected bone resection combined with adjuvant antibiotic-impregnated calcium sulfate and infected bone resection alone, for the treatment of diabetic foot osteomyelitis.
\end{abstract}

Methods: Between 2015 to 2017, 48 limbs (46 patients) with DFO met the criteria were included for assessment. 20 limbs (18 patients) were included in the calcium sulfate group (the CS group) in which vancomycin and/or gentamicinimpregnated calcium sulfate was used as an adjuvant after infected bone resection while 28 limbs (28 patients) as the control group were undergone infected bone resection only. Systemic antibiotics, postoperative wound care and offloading were continued to be applied following surgery in both groups. The time to healing, healing rate, recurrence rate and amputation rate were compared between the two groups.

Results: In total, $90 \%$ (18/20) limbs in the CS group as compared to $78.6 \%(22 / 28)$ infected limbs in the control group went to heal $(P=0.513)$. The Mean time to healing was 13.3 weeks in the CS group and 11.2 weeks in control group $(P=0.132)$. Osteomyelitis recurrence rate was $0 \%(0 / 18)$ in the CS group and $36.4 \%(8 / 22)$ in the control group $(P=0.014)$. Postoperative leakage in calcium sulfate group was $30.0 \%(6 / 20)$ with a mean duration of 8.5 weeks. Amputation rate in the control group was 7.1\% (2/28) compared to 0\% (0/20) in the CS group ( $P=0.153)$.

Conclusions: Antibiotic-impregnated calcium sulfate as an adjuvant prevents the recurrence of DFO but cannot improve the healing rate, reduce the postoperative amputation rate or shorten the time to healing. Prolonged postoperative leakage as the most common complication can be managed with regular dressing.

Level of Evidence: III, Retrospective Comparative Study.

Keywords: Calcium sulfate, Diabetic foot osteomyelitis, Surgical treatment

\footnotetext{
* Correspondence: orthoqin@163.com

${ }^{+}$Cheng-He Qin and Chun-Hao Zhou contributed equally to this work and should both be considered first authors.

'Department of Orthopaedics and Traumatology, Guangdong Second

Provincial General Hospital, Guangzhou 510317, People's Republic of China

Full list of author information is available at the end of the article
}

(c) The Author(s). 2019 Open Access This article is distributed under the terms of the Creative Commons Attribution 4.0 International License (http://creativecommons.org/licenses/by/4.0/), which permits unrestricted use, distribution, and reproduction in any medium, provided you give appropriate credit to the original author(s) and the source, provide a link to the Creative Commons license, and indicate if changes were made. The Creative Commons Public Domain Dedication waiver (http://creativecommons.org/publicdomain/zero/1.0/) applies to the data made available in this article, unless otherwise stated. 


\section{Background}

Diabetic foot osteomyelitis (DFO) is a common complication of patients with diabetic foot infections. It was reported that nearly $20-60 \%$ of patients with diabetic foot might suffer from DFO [1-3]. However, DFO is a difficult-to-treat infection disease, as the treatment of DFO might include the management of chronic ulcers, necrotic soft tissues, gangrenes and of course, the infected bones. Although various treatment methods have been adopted, unfortunately, suffered from DFO still means a high rate of amputation and mortality [4].

Currently, the mainstay treatments for DFO consist of antimicrobial therapy alone or in combination with surgical intervention depending on the severity of disease [5]. In the case of mild infection, antibiotic administration alone for several weeks received promising results [5-7]. However, damaged peripheral vessels condition may make it difficult for parental antimicrobial therapy to achieve satisfying local effects due to insufficient penetration [8]. Moreover, the optimal duration of antibiotic therapy is still controversial with formal studies reported the antibiotic therapy duration varied from 6 weeks to more than 40 weeks $[6,9,10]$. The prolonged duration of antibiotic therapy is limited by the advent of antibiotic-resistant bacteria and potential drug-induced gastrointestinal, liver and kidney injury [11]. For patients with pus, sequestrum, gangrene or antibiotic-resistant bacterial infection [5, 12], surgery is the cornerstone to remove dead tissues and eliminate the infections. For the latest decades, necrotic bones and tissues resection instead of amputation has been widely accepted in treating with DFO, as it removes the infected bones while preserves the healthy bones to minimize the biomechanical changes. However, when carrying out infected bone resection, the completely negative resection margin is relatively difficult to be identified, which may lead to the residue of pathogens. Furthermore, the removal of infected bone sometimes causes the formation of dead space, which will be filled with hematoma soon and provide an environment for the growth of bacteria. Muscle flap used to be a method to obliterate the defects caused by debridement, but it is limited when managing with deeper defects and may disturb the bone healing [13].

Local antibiotic delivery system has been widely used as an adjuvant after the surgical treatment of osteomyelitis and achieved good results $[14,15]$. Compared with intravenous route, the local antibiotic delivery has the advantages of more accurate positioning, higher local concentration, less side effects and longer duration. At the same time, it works as a bone substitute which fills the dead space caused by bone resection and reduces the incidence of reinfection. Polymethyl-methacrylate cement (PMMA) has acted as an antibiotic carrier to fill the defects caused by debridement since Buchholz successfully applied it in joint prosthesis. However, its nonbiodegradable characteristics, the high temperature it produces and a second surgery for removal all limit its application on osteomyelitis especially in DFO [16].

Nowadays, biodegradable antibiotic-impregnated materials such as calcium sulfate, calcium phosphate, bioactive glasses and collagen are gradually applied as a substitute for PMMA in the management of osteomyelitis. All materials mentioned above have advantages of biocompatibility and drug compatibility. Among those substitutes, calcium sulfate is most frequently used materials since it enjoyed some eminent advantages. To begin with, the elution characteristic of loaded antibiotics are now clearly illustrated, an initial burst of antibiotics releasing in the first $24 \mathrm{~h}$ or $48 \mathrm{~h}$ produces antibiotics levels about hundreds to thousands times higher than minimum inhibitory concentration (MIC), then the calcium sulfate releases all antibiotics it loaded gradually at relative slow pace and complete resorption in several weeks. This ideal elution duration makes it more available than collagen (too short) and calcium phosphate (too long) to be a bone graft. Furthermore, it hardly produces the foreign body reaction and helps the formation of new bone. The complications of calcium sulfate are also acceptable, including postoperative drainage and transient hypercalcemia [17].

Previous studies had reported that satisfying outcomes could be received when using antibiotic-impregnated calcium sulfate as an adjuvant after surgical treatment of DFO. However, few comparative studies had been carried out to confirm those results. This retrospective study was designed to observe the outcomes of surgical treatment combined with adjuvant antibiotic-impregnated calcium sulfate versus surgical treatment alone in the treatment of DFO and to compare the differences of healing rate, time to healing, osteomyelitis recurrence rate and amputation rate between two groups.

\section{Methods \\ Participants}

This retrospective study focused on patients with DFO treated in our orthopedic department from January 2015 to June 2017. The main inclusion criteria were as follows: 1) patients with DFO underwent surgical bone resection alone or surgical bone resection combined with adjuvant antibiotic-impregnated calcium sulfate. 2) patients persisted to the follow-up and had been followed for at least 12 months. The main exclusion criteria included: 1) Patients received major amputation or non-surgical treatments. 2) Patients were diagnosed with severe peripheral arterial disease or severe infection according to IDSA. 3) Patients lost to follow up or the follow-up was less than 12 months. Finally, 46 patients with 48 infected limbs met the criteria were included in the study. 


\section{Study design}

Before admitting to our department for surgical treatment, 46 patients (48 limbs) with suspicious DFO (suspected by clinical presentation and the active X-ray, MRI or probeto-bone test results [7] were sampled using percutaneous bone biopsy [18] in our diabetic foot unit for culturing and histology test. Preoperative antibiotic therapy was applied empirically after sampling in the first several days and tailored to culture and susceptibility findings. For patient with negative culture result but accompanied with the presentation of inflammation and positive of histology test, empirical antibiotics were adjusted according to the inflammatory markers. DFO are usually polymicrobial and Staphylococcus aureus has been proven as the most common pathogens in DFO $[19,20]$. Thus, it is a necessity that empirical treatment of DFO should consist of antibiotics with activity against $S$. aureus.

Depending on the calcium sulfate applied or not, 46 patients (48 limbs) were divided into two groups: the CS group and the control group. The characteristics of patients in two groups were presented in Table 1. 20 limbs (18 patients) as the CS group were locally applied with vancomycin and/or gentamicin-impregnated calcium sulfate as an adjuvant after surgical bones resection while 28 limbs (28 patients) as the control group received surgical bones resection only. All surgical procedures were carried out by two experienced surgeons. The surgical treatment performed as the resection of infected bones and removal of the necrotic soft tissues. Healthy bones and soft tissues were preserved as far as possible for minimizing the biomechanical changes and covering the wounds. Once the infected tissues were completely removed, patients in two groups were treated with intravenous antibiotics in 2 weeks individually and followed by oral antibiotics for 4 weeks, according to the recommendation of the International Working Group of Diabetic Foot (IWGDF) [21, 22]. For postoperative wounds care, patients were suggested to offload in involved limbs. Routine dressings and skin moisturizers were applied every two days until wound healing or infection recurring. Once wounds achieving healing, patents were educated never walk in shoes that contributed to a foot ulcer. Customized insoles and shoes were recommended to reduce pressure transfer during follow-up.

In this single-stage study, we defined the wound healing as complete epithelialization covered the wound and the absent of infection. Non-healing was defined if the wound was infected before healing and was treated with a second operation or antibiotics. Osteomyelitis recurrence was defined if the appearance of bone infection was presented at the same or adjacent site after wound healing. Patients suffered from non-healing were excluded from the further calculation for recurrence rate even if the wounds eventually healed with subsequent therapy.

\section{Operative technique}

Surgical procedures were carried out after spinal, nerve block or regional anesthesia. In the CS group, necrotic granulation tissues, pus and infected soft tissues in the

Table 1 Preoperative characteristics of patients in two groups

\begin{tabular}{|c|c|c|c|}
\hline & The CS group & The control group & $P$ value \\
\hline Infected limbs & 20 & 28 & - \\
\hline Age (years) & $59.2(43-76)$ & $61.8(47-83)$ & 0.353 \\
\hline Sex (Male) & 9 & 17 & 0.416 \\
\hline Side (Left) & 9 & 11 & 0.883 \\
\hline Mean duration of DFO (weeks) & $15(1-77)$ & $17(2-257)$ & 0.804 \\
\hline \multicolumn{4}{|l|}{ The Texas classification system } \\
\hline III B & 17 & 21 & \multirow[t]{2}{*}{0.631} \\
\hline$\||| \mathrm{D}$ & 3 & 7 & \\
\hline Hypertension & 8 & 15 & 0.465 \\
\hline Renal insufficiency & 9 & 17 & 0.635 \\
\hline Mean ankle brachial index (ABI) & $1.06(0.82-1.43)$ & $0.98(0.65-1.17)$ & 0.107 \\
\hline Mean WBC count $\left(\times 10^{9} / L\right)$ & $8.09(2.81-14.23)$ & $8.03(3.96-12.07)$ & 0.936 \\
\hline Mean CRP (ng/L) & $32.90(1.17-165.47)$ & $31.93(1.20-114.40)$ & 0.927 \\
\hline Mean ESR (mm/h) & $86(34-134)$ & $88(30-140)$ & 0.829 \\
\hline Mean Albumin $(\mathrm{g} / \mathrm{L})$ & $33.3(20.9-42.6)$ & $31.8(24.7-36.9)$ & 0.304 \\
\hline Mean Creatinine $(\mu \mathrm{mol} / \mathrm{L})$ & $135(38-682)$ & $113(33-513)$ & 0.586 \\
\hline Mean HbA1c (\%) & $8.6(5.6-10.1)$ & $8.2(4.8-11.2)$ & 0.768 \\
\hline
\end{tabular}

Abbreviations: CS Calcium sulfate, DFO Diabetic foot osteomyelitis, WBC white blood cell, ESR Erythrocyte sedimentation rate, HBA1c Glycosylated hemoglobin A1c 
ulcers were removed until the bleeding tissue has been exposed. Following the removal of ulcers or sinus, the bone procedures were carried out. If osteomyelitis was located in diaphyses, devitalized bones in the base of ulcers were exposed and excised to the level of healthy cancellous and cortical bone. An extra $2 \mathrm{~mm}$ of healthy bone was also resected in the prophylaxis of residual pathogens. If possible, the bases of metatarsal and phalangeal bones were necessary to be preserved for healthy tendons attaching. When infections were located in interphalangeal or metatarsophalangeal joints, however, the joints as well as partial distal and proximal bones were needed to be excised. Fibrous tissues, fascia and tendons nearby were also completely removed in case the residue of pathogens. Following bones resection, the defects were irrigated with $0.05 \%$ chlorhexidine solution and sterile saline solution. If necessary, Kirschner wires were adopted to maintain the bones stable. After removal of infected bones and necrotic soft tissues, antibiotic-impregnated calcium sulfate was prepared. Vancomycin and/or gentamicin was mixed into the synthetic calcium sulfate (Stimulan, Biocomposite Ltd., UK) with a recommended ratio: $0.5 \mathrm{~g}$ vancomycin with $5 \mathrm{ml}$ calcium sulfate or $80 \mathrm{mg}$ gentamicin with $5 \mathrm{ml}$ calcium sulfate. Then they were dissolved with sterile saline solution and injected into the dead space (range from $0.5 \mathrm{ml}$ to $5 \mathrm{ml}$ individually). After operations, the wounds were sutured primarily without tension. In the control group, patients received the same operation expect for the application of antibiotic-loaded calcium sulfate.

\section{Statistical analysis}

Data were collated using Microsoft Excel (Redmond, Washington) and analyzed using SPSS v20 (SSPS Inc., Chicago, Illinois). Continuous variables which were verified of normal distribution and the homogeneity of variance were compared using Independent-Samples $\mathrm{T}$ Test; Continuous variables which failed to pass normality test were compared using a Mann-Whitney $U$ test. Pearson $\mathrm{X}$ 2, Continuity Correction Chi-square Test or Fisher Exact Test were used in comparing the demographic data, healing rate, recurrence rate and amputation rate. $P<0.05$ was considered statistically significant.

\section{Results}

From 2015 to 2017, 46 patients (48 limbs) met the criteria were included in the study. The locations of DFO were 16 in phalanges (7 in the CS group), 13 in metatarsal bones ( 5 in the CS group) and 19 in both phalanges and metatarsal bones (8 in the CS group).

The preoperative culture results were presented in Table 2 . 95.0\% (19/20) samples in the CS group showed positive culture results with total of 24 bacterial spices isolated. In control group, 89.3\% (25/28) samples were culture-positive with 40 isolated bacterial spices. $35.0 \%(7 / 20)$ infected limbs in the CS group were monomicrobial infections compared to $42.9 \%(12 / 28)$ monomicrobial infections in the control group. Staphylococcus aureus was the most common pathogen isolated by culture followed by Escherichia coli and Enterococcus faecalis.

Twenty limbs (41.7\%) as the CS group were applied with antibiotic calcium sulfate after infected bone resection compared to 28 limbs (58.3\%) as the control group underwent infected bone resection alone. The follow-up outcomes of two groups were presented in Table 3 . During follow up, 2 patients in the CS group died of cardiovascular disease after surgery, but the wounds had healed before death and did not recur within 1 year after surgery. 2 patients applied antibiotic-impregnated CS are presented in Figs. 1 and 2.

\section{Discussion}

According to the severity of infections and local blood supply, diabetic foot osteomyelitis can be managed with conservative treatment or surgery. When accompanied with pus, substantial bone necrosis, gangrene, recurrent ulcer or antibiotic-resistant bacteria infection, surgery is recommended to remove necrosis tissues, reduce the antibiotic therapy duration and correct bone deformity to promote healing [23]. However, even surgical treatment also has its own limitations. Due to the bone removal, biomechanics in foot is inevitably changed, which may lead to the ulcer formation in a new position. Thus, postoperative offloading with customized

Table 2 The microbiological findings in two groups

\begin{tabular}{lll}
\hline microbiological findings & The CS group & The control group \\
\hline Monomicrobial infections & $7(35.0 \%)$ & $12(42.9 \%)$ \\
Total bacterial spices & 24 & 39 \\
Staphylococcus aureus & 8 & 8 \\
MRSA & 1 & 0 \\
Escherichia coli & 2 & 8 \\
Enterococcus faecalis & 2 & 5 \\
Proteus species & 2 & 3 \\
Klebsiella species & 2 & 1 \\
Pseudomonas aeruginosa & 1 & 3 \\
Staphylococcus epidermidis & 1 & 0 \\
Streptococcus species & 2 & 2 \\
Acinetobacter baumannii & 0 & 2 \\
Candida albicans & 0 & 2 \\
Enterobacter cloacae & 0 & 1 \\
Stenotrophomonas maltophilia & 1 & 0 \\
Myroides odoratimimus & 1 & 0 \\
Negative finding & 1 & 4
\end{tabular}

Abbreviations: CS Calcium sulfate, MASA Methicillin resistantstaphylococcus aureus 
Table 3 The follow-up outcomes of two groups

\begin{tabular}{llll}
\hline & The CS group & The control group & $P$ value \\
\hline Mean hospital stay (days) & $24.8(7-59)$ & $28.3(9-56)$ & 0.341 \\
Mean follow-up duration(months) & $17.6(12-38)$ & $20.1(12-30)$ & $15.6(6-28)$ \\
Preoperative antibiotics duration (days) & $13.5(4-30)$ & $9.8(2-14)$ & 0.120 \\
Postoperative IV antibiotics duration (days) & $8.8(3-14)$ & $78.6 \%(22 / 28)$ & 0.142 \\
Postoperative healing rate & $90.0 \%(18 / 20)$ & $11.2(2-26)$ & 0.569 \\
Mean healing duration (weeks) & $13.3(5-30)$ & $36.4 \%(8 / 22)$ & - \\
Recurrence rate & $0.0 \%(0 / 18)$ & - & -132 \\
Postoperative leakage rate & $30 \%(6 / 20)$ & - & - \\
Postoperative leakage duration (weeks) & $8.5(4-13)$ & $7.1 \%(2 / 28)$ & 0.13 \\
Amputation rate & $0.0 \%(0 / 20)$ & -
\end{tabular}

insoles and shoes was essential to promote healing and prevent infection recurrence. Furthermore, during infected bone resection, the clear margin of bone and soft tissue is fairly difficult to identify. The exact extent for infected bone resection is largely depended on the intra-operative judgement of the surgeons and thus sometimes lead to the non-healing or recurrence of osteomyelitis. To eradicate the residual infection, local antibiotic-impregnated calcium sulfate was applied in our study as the high local antibiotic level it produced.
In total, $90.0 \%(18 / 20)$ limbs in CS group and $78.6 \%$ $(22 / 28)$ limbs in control group healed after the first operation. The higher healing rate in CS group is in accordance with the retrospective study of Rajesh $M$. Jogia et al., who reported all DFO limbs (20 patients) achieved healing after surgical debridement combining with antibiotic-impregnated calcium sulfate beads application [24]. Similarly, Noman Shakeel Niazia et al. retrospectively studied 70 patients with DFO who received debridement and adjunctive antibiotic-loaded calcium

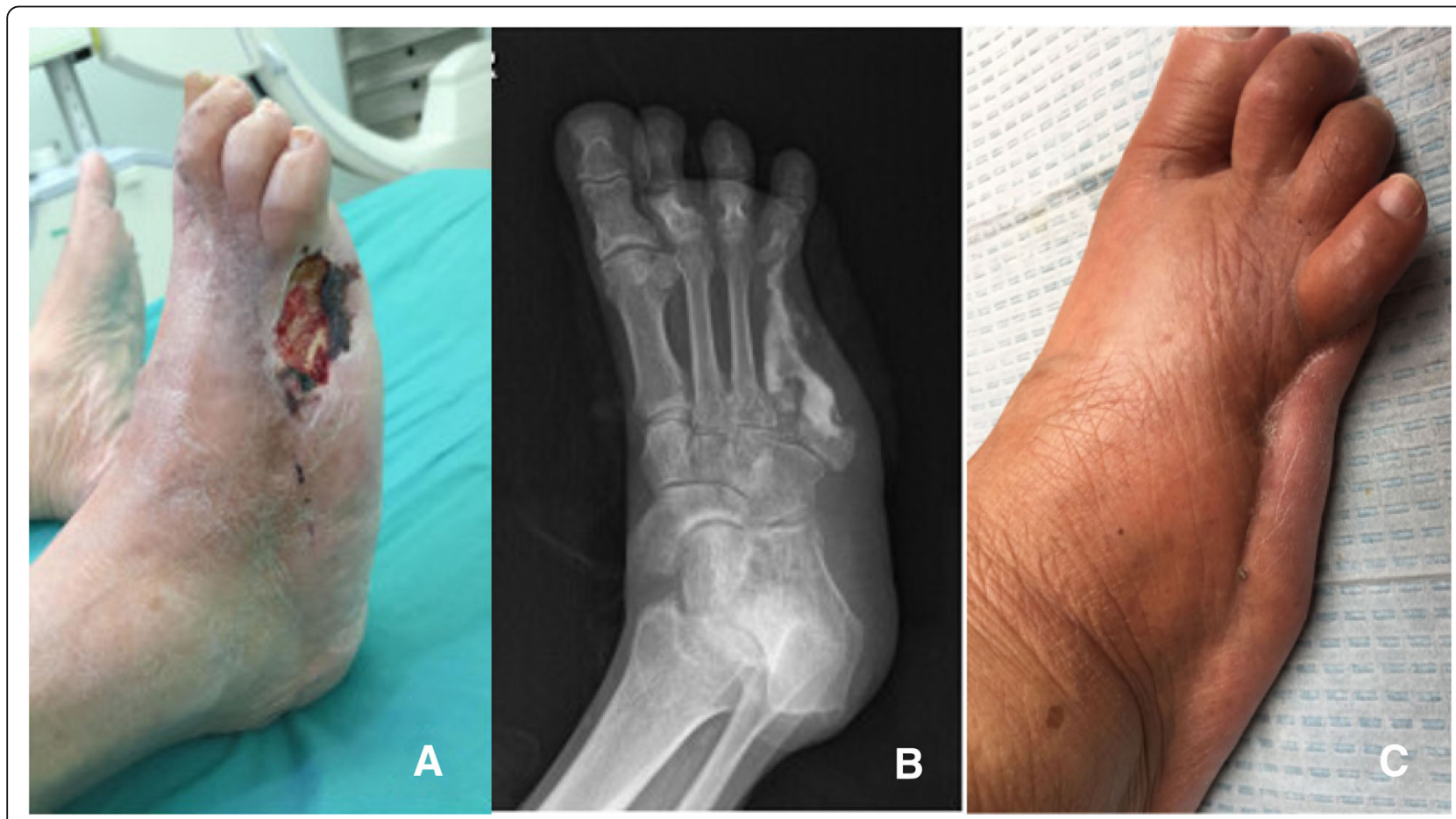

Fig. 1 A patient with the fourth infected metatarsal (the fifth phalanx and metatarsal had been resected 5 years ago) were resected to the base of metatarsal before the vancomycin-impregnated calcium sulfate was injected into the dead space. a The presentation of wound before the operation. $\mathbf{b}$ The X-ray presentation 3 days after operation. $\mathbf{c}$ The ulcer had healed and no symptoms of osteomyelitis were presented 1 year after operation 

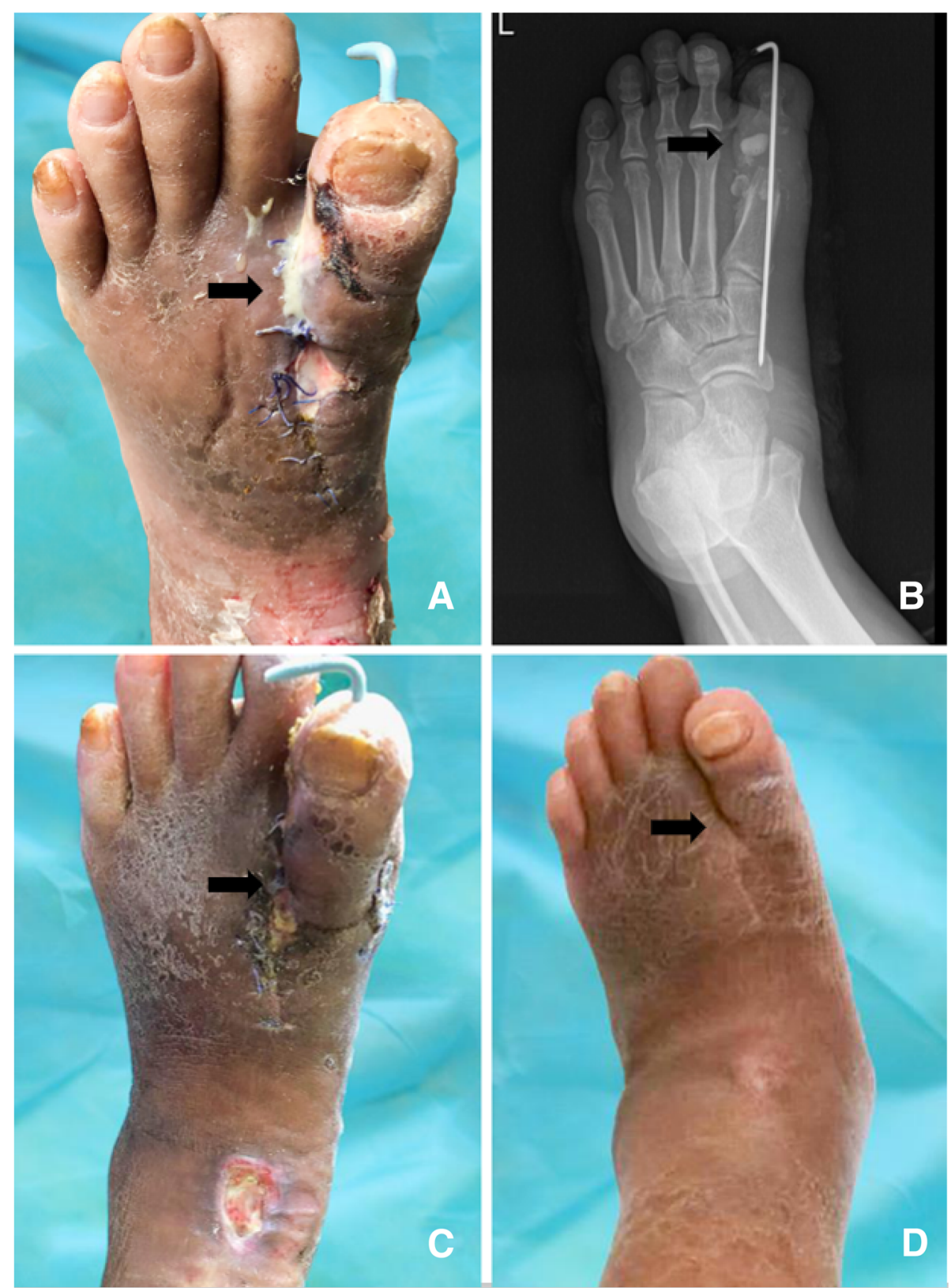

Fig. 2 A 62-yaer-old patient with DFO on left first metatarsophalangeal joint. The metatarsophalangeal joint, partial metatarsal and phalange were removed. a The postoperative leakage of calcium sulfate. This sterile leakage was demonstrated as a kind of white, foamy, antibiotics-containing fluid. $\mathbf{b}$ X-ray presentation. Vancomycin-impregnated calcium sulfate lump was degrading. $\mathbf{c} 7$ weeks after operation, the skin needed to be moistened. Superficial ulcer in ankle could be managed with dressing. $\mathbf{d} 1$ year after surgical treatment. Although edema was presented, the operative wound healed and the symptoms of infection were disappeared

sulfate treatment. During an average follow-up of 10 months, infection eradication and wound healing were achieved in 90 and $81 \%$ limbs respectively [25]. Proper explanation of the high healing rate is that much higher antibiotic concentration reached topically can eradicate more residual organisms with the resorption of the calcium sulfate. Former studies have shown antibiotic levels surpass 200 times the MIC [14] for organisms over days and still retain antimicrobial effect after 6 weeks to 3 months [26, 27], which is sufficient to penetrate the biofilm and eradicate the residual organisms. Unfortunately, a significant difference in the healing rate between the two groups was not found through statistical analysis (90\% in CS group versus $78.6 \%$ in control group, $P>0.05)$. Combining with the previously-reported efficacy of antibiotic-impregnated calcium sulfate in eradicating 
infections and well-controlled variables (similar blood supply condition, appropriate wound care and postoperative offloading between two groups) in our study, we deem that the small group of patients included may cause the absence of significant differences in the healing rate. This explains why the healing rates of two groups are not statistically significant. With regard to the recurrence rate, such high topical antibiotic concentration and long therapeutic duration explained that the much lower recurrence rate of antibiotic-impregnated calcium sulfate group than the control group. Rajesh M. Jogia et al. reported no recurrence in 20 patients who received surgical debridement combined with gentamicin or vancomycin-impregnated calcium sulfate beads within the follow-up of 12 months after surgical intervention, which is similar with the result in our study $[26,27]$.

However, our study failed to provide evidence that antibiotic-impregnated calcium sulfate will shorten the wound healing duration, which is different from the similar former study. Martin Varga et al. reported that application of gentamicin-impregnated collagen sponge shortened nearly 2 weeks of wound healing duration after minor amputation [28]. Fabian G. Krause et al. found that time to a dry wound was 5.2 weeks in the antibiotic-impregnated beads group and 7.0 weeks in the control group, even if no significance in two groups [29]. In this study, however, the mean duration of healing in CS group was 2.1 weeks longer than the mean healing duration in control group. Actually, we hypothesize that the prolonged leakage in CS group may interfere with the wound healing, but no previous study was found to support this hypothesis.

Prolonged postoperative leakage was found to be the most common complication in calcium sulfate treated patients. $30.0 \%$ limbs $(6 / 20)$ suffered from prolonged postoperative leakage in CS group with a mean duration of 8.5 weeks. With regular dressing, all wounds achieved healing eventually. The drainage rate is similar with the former studies about chronic osteomyelitis and varies from 4.2 to $32 \%[13,17,30]$. Other studies have reported the prolonged postoperative leakage in treating chronic osteomyelitis after using antibiotic-impregnated calcium sulfate, but achieving healing with appropriate wound care $[13,31]$. In fact, prolonged postoperative leakage itself is neither an indication for a second surgery nor did it relate to reinfection of the wound [31]. Regularly dressing in outpatient is enough in dealing with the postoperative leakage. Vacuum-assisted Closure (VAC) was not used because we deemed that it might lower the topical antibiotic level when pumping the drainage. During surgical treatment, good soft tissue coverage and primary closure are essential methods in the prevention of postoperative leakage.

Severe side effects were not found excluding postoperative leakage in CS group. The explanation is that the dose of vancomycin or gentamicin administered locally was less than $0.5 \mathrm{~g}$ (vancomycin) or $100 \mathrm{mg}$ (gentamicin), which means the systemic concentration reached was much lower than the same dose applied intravenously because of the slow releasing of antibiotics with the degradation of calcium sulfate. Unfortunately, systemic drug concentration was not obtained to confirm our hypothesis. In their study, Zhang et al. measured the blood vancomycin levels in 24 osteomyelitis patients locally applied with vancomycin-impregnated calcium sulfate beads (range from $1.5 \mathrm{ml}$ to $5 \mathrm{ml}$ with a ratio of $1 \mathrm{~g}$ vancomycin: $5 \mathrm{ml}$ calcium sulfate). The results showed that the mean blood vancomycin level was still within a safe range for application [32]. P. Wahl et al. found that even $6 \mathrm{~g}$ vancomycin was applied locally, the systemic concentration remained within a safe range and local concentration was still below the reported cell toxicity thresholds [27].

To our knowledge, our study is the first retrospective comparative study comparing the outcomes of infected bone resection combined with adjunctive antibiotic-impregnated calcium sulfate versus infected bone resection in the treatment of DFO. The limitations of our study are mainly in two aspects. Firstly, the follow-up duration in two groups may not be enough to show the outcomes of all patients, which may influence the healing rate, recurrence rate and amputation rate in our study. Furthermore, it is a retrospective study for forefoot DFO with a small group of patients, the additional studies are necessary to confirm our findings.

\section{Conclusion}

Application of the antibiotic-impregnated calcium sulfate as an adjuvant can be regarded as efficacious for preventing the recurrence of forefoot DFO. However, evidence is not found that the use of antibiotic-impregnated calcium sulfate improves the healing rate, shorten the healing duration or reduce the amputation rate. Prolonged postoperative leakage as a common complication can be dealt with regular dressing.

\section{Abbreviations \\ CRP: C-reactive Protein; CS: Calcium Sulfate; DFO: Diabetic Forefoot Osteomyelitis; ESR: Erythrocyte Sedimentation Rate; IDSA: Infectious Diseases Society of America; IWGDF: International Working Group of Diabetic Foot; MIC: Minimum Inhibitory Concentration; MRI: Magnetic Resonance Imaging; PMMA: Polymethyl-methacrylate cement; VAC: Vacuum-assisted Closure}

Acknowledgements

We would like to thank all the people who helped us in the current study.

\section{Funding}

This study was not externally founded.

Availability of data and materials

The data used and analyzed during the current study is available from the corresponding author on reasonable request. 


\section{Authors' contributions}

$\mathrm{CHQ}$ and $\mathrm{CHZ}$ contributed equally to this work. Scientific idea: $\mathrm{CHQ}, \mathrm{CHZ}$; Project planning: $\mathrm{CHQ}, \mathrm{CHZ}, \mathrm{HJS}, \mathrm{GYC}, \mathrm{HAZ}$, JF RT; Manuscript writing: $\mathrm{CHQ}$, CHZ; Manuscript revision: $\mathrm{CHZ}, \mathrm{HJS}, \mathrm{GYC}, \mathrm{HAZ}, \mathrm{JF}$, RT; All authors read and approved the final manuscript.

\section{Ethics approval and consent to participate}

Medical Ethical Committee of Nanfang Hospital of Southern Medical University has approved the Research ethics approval. All included patients consented to participate in this study and a signed consent form was obtained from each subject before testing. All procedures were conducted according to the Declaration of Helsinki.

\section{Consent for publication}

Not applicable.

\section{Competing interests}

The authors declare that they have no competing interests.

\section{Publisher's Note}

Springer Nature remains neutral with regard to jurisdictional claims in published maps and institutional affiliations.

\section{Author details}

'Department of Orthopaedics and Traumatology, Guangdong Second Provincial General Hospital, Guangzhou 510317, People's Republic of China. ${ }^{2}$ Department of Orthopaedics and Traumatology, Provincial Key Laboratory of Bone and Cartilage Regenerative Medicine, Nanfang Hospital, Southern Medical University, Guangzhou 510515, People's Republic of China. ${ }^{3}$ Department of Nursing, Nanfang Hospital, Southern Medical University, Guangzhou 510515, People's Republic of China.

Received: 15 February 2019 Accepted: 16 May 2019

Published online: 24 May 2019

\section{References}

1. Lavery LA, Peters EJ, Armstrong DG, Wendel CS, Murdoch DP, Lipsky BA. Risk factors for developing osteomyelitis in patients with diabetic foot wounds. Diabetes Res Clin Pract. 2009;83(3):347-52.

2. Shone A, Burnside J, Chipchase S, Game F, Jeffcoate W. Probing the validity of the probe-to-bone test in the diagnosis of osteomyelitis of the foot in diabetes. Diabetes Care. 2006;29(4):945.

3. Senneville E, Robineau O. Treatment options for diabetic foot osteomyelitis. Expert Opin Pharmaco. 2017;18(8):759-65.

4. Lavery LA, Armstrong DG, Wunderlich RP, Mohler MJ, Wendel CS, Lipsky BA Risk factors for foot infections in individuals with diabetes. Diabetes Care. 2006:29(6):1288-93.

5. Lipsky BA. Treating diabetic foot osteomyelitis primarily with surgery or antibiotics: have we answered the question? Diabetes Care. 2014;37(3):593-5

6. Embil JM, Rose G, Trepman E, Math MC, Duerksen F, Simonsen JN, Nicolle LE. Oral antimicrobial therapy for diabetic foot osteomyelitis. Foot Ankle Int. 2006;27(10):771-9.

7. Lázaro-Martínez JL, Aragón-Sánchez J, García-Morales E. Antibiotics versus conservative surgery for treating diabetic foot osteomyelitis: a randomized comparative trial. Diabetes Care. 2014;37(3):789-95.

8. Hajdu S, Lassnigg A, Graninger W, Hirschl AM, Presterl E. Effects of vancomycin, daptomycin, fosfomycin, tigecycline, and ceftriaxone on Staphylococcus epidermidis biofilms. J Orthop Res. 2009;27(10):1361-5.

9. Valabhji J, Oliver N, Samarasinghe D, Mali T, Gibbs RG, Gedroyc WM. Conservative management of diabetic forefoot ulceration complicated by underlying osteomyelitis: the benefits of magnetic resonance imaging Diabet Med. 2009:26(11):1127-34

10. Tone A, Nguyen S, Devemy F, Topolinski H, Valette M, Cazaubiel M, Fayard A, Beltrand E, Lemaire C, Senneville E. Six-week versus twelve-week antibiotic therapy for nonsurgically treated diabetic foot osteomyelitis: a multicenter open-label controlled randomized study. Diabetes Care. 2015; 38:302-7 DIABETES CARE 2015, 38(4):735.

11. van Asten S, Mithani M, Peters E, La Fontaine J, Kim PJ, Lavery LA. Complications during the treatment of diabetic foot osteomyelitis. Diabetes Res Clin Pract. 2017;135:58-64.
12. Aragon-Sanchez J, Lipsky BA. Modern management of diabetic foot osteomyelitis. The when, how and why of conservative approaches. Expert Rev Anti-Infect Ther. 2018;16(1):35-50.

13. Ferguson JY, Dudareva M, Riley ND, Stubbs D, Atkins BL, McNally MA. The use of a biodegradable antibiotic-loaded calcium sulphate carrier containing tobramycin for the treatment of chronic osteomyelitis: a series of 195 cases. Bone Joint J. 2014;96-B(6):829-36.

14. Gauland C. Managing lower-extremity osteomyelitis locally with surgical debridement and synthetic calcium sulfate antibiotic tablets. Adv Skin Wound Care. 2011;24(11):515-23.

15. Branstetter JG, Jackson SR, Haggard WO, Richelsoph KC, Wenke JC. Locallyadministered antibiotics in wounds in a limb. J Bone Joint Surg Br. 2009; 91(8):1106-9.

16. McNally MA, Ferguson JY, Lau AC, Diefenbeck M, Scarborough M, Ramsden A, Atkins BL. Single-stage treatment of chronic osteomyelitis with a new absorbable, gentamicin-loaded, calcium sulphate/hydroxyapatite biocomposite: a prospective series of 100 cases. Bone Joint J. 2016;98-B(9):1289-96.

17. Kallala R, Harris WE, Ibrahim M, Dipane M, McPherson E. Use of Stimulan absorbable calcium sulphate beads in revision lower limb arthroplasty: safety profile and complication rates. Bone Joint Res. 2018;7(10):570-9.

18. Senneville E, Melliez H, Beltrand E, Legout L, Valette M, Cazaubiel M, Cordonnier M, Caillaux M, Yazdanpanah Y, Mouton Y. Culture of percutaneous bone biopsy specimens for diagnosis of diabetic foot osteomyelitis: concordance with ulcer swab cultures. Clin Infect Dis. 2006;42(1):57-62.

19. van Asten SAV, La Fontaine J, Peters EJG, Bhavan K, Kim PJ, Lavery LA. The microbiome of diabetic foot osteomyelitis. Eur J Clin Microbiol. 2016;35(2):293-8.

20. Lavigne J, Sotto A. Microbial management of diabetic foot osteomyelitis. Future Microbiol. 2017;12(14):1243-6.

21. Lipsky BA, Berendt AR, Cornia PB, Pile JC, Peters EJ, Armstrong DG, Deery HG, Embil JM, Joseph WS, Karchmer AW, et al. 2012 Infectious Diseases Society of America clinical practice guideline for the diagnosis and treatment of diabetic foot infections. Clin Infect Dis. 2012;54(12):e132-73.

22. Lipsky BA, Aragón-Sánchez J, Diggle M, Embil J, Kono S, Lavery L, Senneville É, Urbančič-Rovan V, Van Asten S, Peters EJG. IWGDF guidance on the diagnosis and management of foot infections in persons with diabetes. Diabetes Metab Res Rev. 2016;32:45-74.

23. Biz C, Gastaldo S, Dalmau-Pastor M, Corradin M, Volpin A, Ruggieri P. Minimally invasive distal metatarsal Diaphyseal osteotomy (DMDO) for chronic plantar diabetic foot ulcers. Foot Ankle Int. 2017;39(1):83-92.

24. Jogia RM, Modha DE, Nisal K, Berrington R, Kong MF. Use of highly purified synthetic calcium sulfate impregnated with antibiotics for the management of diabetic foot ulcers complicated by osteomyelitis. Diabetes Care. 2015:38(5):e79-80.

25. Niazi NS, Drampalos E, Morrissey N, Jahangir N, Wee A, Pillai A. Adjuvant antibiotic loaded bio composite in the management of diabetic foot osteomyelitis - a multicentre study. Foot. 2019;39:22-7.

26. Aiken SS, Cooper JJ, Florance H, Robinson MT, Michell S. Local release of antibiotics for surgical site infection management using high-purity calcium sulfate: an in vitro elution study. Surg Infect. 2015;16(1):54-61.

27. Wahl P, Guidi M, Benninger E, Rönn K, Gautier E, Buclin T, Magnin JL, Livio F. The levels of vancomycin in the blood and the wound after the local treatment of bone and soft-tissue infection with antibiotic-loaded calcium sulphate as carrier material. Bone Joint J. 2017;99-B(11):1537-44.

28. Varga M, Sixta B, Bem R, Matia I, Jirkovska A, Adamec M. Application of gentamicin-collagen sponge shortened wound healing time after minor amputations in diabetic patients - a prospective, randomised trial. Arch Med Sci. 2014;10(2):283-7.

29. Krause FG, DeVries G, Meakin C, Kalla TP, Younger AS. Outcome of transmetatarsal amputations in diabetics using antibiotic beads. Foot Ankle Int. 2009;30(6):486-93.

30. Alrashidi $Y$, Hügle T, Wiewiorski M, Herrera-Perez M, Valderrabano V. Surgical treatment options for the diabetic Charcot midfoot deformity. Clin Podiatr Med Sur. 2017;34(1):43-51.

31. Drampalos E, Mohammad HR, Kosmidis C, Balal M, Wong J, Pillai A. Single stage treatment of diabetic calcaneal osteomyelitis with an absorbable gentamicin-loaded calcium sulphate/hydroxyapatite biocomposite: the Silo technique. Foot. 2018;34:40-4.

32. Zhang Z, Zhang C, Guo QF, Shen LF, Zhang XW. Application of vancomycin-loaded calcium sulphate in treatment of osteomyelitis. Zhongguo Yi Xue Ke Xue Yuan Xue Bao. 2013;35(3):337-42. 